\title{
Repeatability of the timed 25-foot walk test for individuals with multiple sclerosis
}

Clinical Rehabilitation

27(8) 719-723

(C) The Author(s) 2013

Reprints and permissions: sagepub.co.uk/journalsPermissions.nav DOI: I0.1 I77/02692/55|2470269 cre.sagepub.com

@SAGE

\section{Rebecca D Larson',2, Daniel J Larson 1,2, Ted B Baumgartner' and Lesley J White'}

\begin{abstract}
Objective: The purpose of this study was to determine if there is a practice effect present in the timed 25 -foot walk in ambulatory individuals with multiple sclerosis.

Design: Thirty six people (30 women and 6 men) diagnosed with relapsing remitting multiple sclerosis participated in two testing sessions, one week apart. Each participant performed two sequential trials of the timed 25-foot walk test per session and the walk performance was measured with a laser timing system.

Results: We observed improvements in walking speed between the two trials of session one (trial one: $6.42(0.09)$ vs. trail two: $5.97(0.08)$ seconds, $p<0.001)$. Within session two, performance remained stable (trial three: $5.7 \mathrm{I}(0.07)$ vs. trial four: $5.63(0.07)$ seconds, $p>0.05)$. We also observed a significant improvement in walking speed when averages of the two trials were compared across sessions (session I: 6.19 (0.09) vs. session 2: $5.67(0.07)$ seconds, $p<0.0$ I).

Conclusions: Based on our results, familiarization of the timed 25-foot walk test improves stability of walk performance scores in ambulatory individuals with relapsing remitting multiple sclerosis.
\end{abstract}

\section{Keywords}

Multiple sclerosis, assessment, clinical tests, mobility, repeatability

Received: 19 June 2012; accepted: 10 November 2012

\section{Introduction}

A host of innovative diagnostic and disease monitoring methods have been used to assess disability and responses to targeted therapies for individuals with multiple sclerosis (MS). One of the most commonly used measures of disability is the Expanded Disability Status Scale (EDSS). ${ }^{1}$ While comprehensive, the EDSS score has been criticized by some for suboptimal inter-rater reliability, marginal sensitivity to change in disability, a bias towards locomotor function, ${ }^{2-5}$ as well as the need for a trained neurologist for valid scoring. ${ }^{2}$ In some instances the timed 25-foot walk test has been used alone to assess disease progression ${ }^{6}$ as opposed to the EDSS score.

'Department of Kinesiology, University of Georgia, Athens, GA, USA

2Department of Health and Exercise Science, University of Oklahoma, Norman, OK, USA

Corresponding author:

Rebecca D Larson, Health and Exercise Science, University of Oklahoma, Norman, OK 73019, USA.

Email: rdlarson@ou.edu 
The National Multiple Sclerosis Society appointed Task Force on Clinical Outcomes Assessment recommended a new approach in assessing clinical outcome measures, which lead to the development of the Multiple Sclerosis Functional Composite (MSFC) score. ${ }^{7-9}$ The MSFC test includes quantitative assessments of arms/hand function (Nine-hole Peg Test), cognitive function (Three-second Paced Auditory Serial Test) and leg function/ambulation (timed 25-foot walk test)) with performance scores averaged and transformed into $z$ scores for comparison purposes. ${ }^{7,9,10}$ There appears to be a practice effect of the MSFC as evidenced by Cohen and colleagues and Fischer et al. that lead to the recommendation that the MSFC test should be administered with a pre-baseline testing session to familiarize the subjects and examiners with the testing protocol., 911 Despite these recommendations, test administration procedures have been inconsistent, with some researchers using only one or no pre-baseline measures. ${ }^{12-21}$ The lack of consistency in testing procedures provides the possibility of biasing test results, such that the change in walk speed might not arise from the intervention or treatment, but rather owing to a practice effect. Therefore, the purpose of this study was to determine if there is a significant practice effect present in the timed 25-foot walk test.

\section{Methods}

\section{Participants}

Thirty six volunteers (30 females and 6 males) with clinically stable relapsing remitting MS signed a written informed consent approved by the University Institutional Review Board prior to study enrollment. Participants had an EDSS score $<6.5$ (able to walk at least one city block), no orthopedic limitations or other health related issues that would preclude safe participation and no prior exposure to the test procedures. ${ }^{1}$

\section{Study design and timed 25-foot walk procedures}

This study involved making repeated measurements on two testing occasions. Study participants performed a total of four trials of the timed 25-foot walk test.
Two sequential trials were performed during the initial test session and two trials were performed again, one week later, during a second test session. A digital timing system (Brower IRD-T175, Salt Lake City, Utah) was used to assess walk performance. The timing system included start and finish laser sensors that were positioned 91.4 centimeters (36 inches) apart, which is the width of a common doorway) and 38 centimeters (15 inches) from the ground. All participants started in a standardized standing "start position", which was 12.7 centimeters ( 5 inches) behind the laser timing system. The timing system started and stopped when the participant broke the laser sensor's plane. Each participant received standard instructions that they all understood: "I'd like you to walk 25 feet as quickly as possible, but safely. Do not slow down until after you've passed the finish-line. Ready? Go". The task was immediately administered for a second trial in the same manner as the first trial. The participants returned within one week at approximately the same time of day and performed the same test as before (session two). The recommended procedures for scoring the timed 25 -foot walk test are to take the average of two completed trials. ${ }^{10}$ The participants were at no time advised of their performance scores from trial to trial.

\section{Data analysis}

All analyses were performed using SPSS software (SPSS Inc., Version 16. Chicago, IL). Repeated measures analysis of variance (ANOVA) were used to detect differences across the four trials (two trails on each of the two sessions) of the timed 25 -foot walk test. Dependent $t$-tests were then used to compare differences between trials if the $F$-test was significant. An alpha of 0.05 was used to establish statistical significance. Additionally an intraclass correlation coefficient was calculated to assess test-retest reliability. To obtain the mean square values each participant had an average score for session one (trial one and trial two) and for session two (trial three and trial four).

\section{Results}

A total of 36 volunteers performed the timed 25 foot walk test (30 females and 6 males, 12 participants 
had EDSS scores $>4.5$ and two participants used walking aids). Participants had a mean age, height, weight, body mass index and EDSS score of 45.2 (1.8) years, 1.7 (0.02) meters, 82.4 (3.4) kilograms, $28.4(1.0) \mathrm{kg} / \mathrm{m}^{2}$, and $3.5(0.4)$, respectively. Additionally they had low levels of physical activity with no more than two times per week of 15-30 minutes of low to moderate intensity activities. Average walk times were significantly faster on trial two than on trial one (trial one: $6.42(0.09)$ seconds vs. trial two: 5.97 (0.08) seconds, $p<0.001)$. However, no significant differences were observed between trials two, three and four (trial two: 5.97 (0.08) seconds vs. trial three: 5.7 (0.07) seconds, $p$ $>0.05$ ); (trail three: $5.71(0.07)$ seconds vs. trial four: $5.63(0.07)$ seconds, $p>0.05)$.

When trials within each session were averaged, we observed significantly faster walk times on the second testing session (session one: 6.19 (0.09) seconds vs. session two: $5.67(0.07)$ seconds, $p<0.01)$. The intraclass correlation coefficient (reliability) for the average of the two trials within a session was 0.92 .

\section{Discussion}

The main findings of this study were: 1) significant improvements in timed 25-foot walk performance across the first two trials that were stabilized by the third trial; and 2) when using the current MSFC score recommendations of administering two trials and averaging the times, we observed significantly faster gait speeds during the second test session. Our findings are consistent with other reports that suggest that improvements in timed 25-foot walk times might be associated with a practice effect. ${ }^{9}$ However, others have reported non-significant improvements in walk times during practice trials. ${ }^{22-23}$ Possible reasons for the disparate findings may include: 1) participant's prior exposure to testing procedures; 2) participant variability (disease type and disability status) of symptoms; and/or 3) variability in researchers administrating the timed 25-foot walk test. Participants in our study reported no prior experience with the walk test procedures, which may help explain some of the discrepant findings between our results and other investigators, as practice has been shown to improve performance. ${ }^{11}$ Additionally, the observed practice effect seemed to persist over a one-week period. Some of our unpublished preliminary data further indicates that this practice effects persists after as long as six months.

The disability range of the participants in this study may help explain discrepancies in study outcomes. Our study was limited to people with relapsing remitting MS and an EDSS score of less than 6.5. Reliability studies of the MSFC scores and the timed 25-foot walk test include a variety of participants with EDSS scores ranging from 0-8.0 and diverse types of disease status (relapsing remitting, primary progressive and secondary progressive). ${ }^{9-11,24}$ The variability of samples could account for differences within and between studies. One way to determine test-retest reliability is through an intraclass correlation coefficient. At first glance, it appears that our data has very good reliability between sessions, $R=0.92$. However, owing to the high variability between subjects, our reliability coefficient may have been inflated.

Finally, differences in administration and/or scoring of walking trials may contribute to dissimilar findings between studies. For example, the recommended procedures for the MSFC is to administer the timed 25-foot walk test twice and reporting the average of the two trials, with no mention of a practice session, despite reference to reliability studies indicating practice session recommendations. ${ }^{9,11}$

In some instances, the lack of methodological details about test administration procedures makes it difficult to discern whether the results being reported reflect an individual's true change in performance ability. When comparing test administration procedures across studies, specific details of testing methods are not always described, leaving it unclear whether a practice session occurred or whether participants had previous experience with the test. ${ }^{11,22,23,25}$ In some instances, the timed 25-foot walk test was administered with one trial in a session, ${ }^{19}$ whereas in another study, the test was performed four times and the scores were averaged. ${ }^{20}$ These variations in test administration procedures could account for some of the different findings observed between studies. 
Another complicating factor for the proper interpretation of timed 25-foot walk test performance arises from the MSFC itself. The MSFC was originally developed utilizing $z$ scores wherein an individual performance score $(x)$ minus the mean of the group $(y)$ is divided is by standard deviation (SD) $[(\mathrm{x}-\mathrm{y}) /(\mathrm{SD})]$. There have been reliability and validity studies to assess the impact the practice effects have on $z$ scores. ${ }^{9,22,25}$ In regards to the timed 25-foot walk test, the learning effect was found to be minimal when using a $z$ scores. This transformation could result in underreporting the absolute magnitude of the variability measures. For example, if an individual had a score of $x=7$ seconds on trial 1 of the timed 25-foot walk test and the group mean of $y$ $=5$ seconds and $\mathrm{SD}=2$, the calculated $z$ score is 1.0. If that same individual was retested and his/her walk time slowed to $X=10$ seconds and the group mean shifted to $Y=8$ seconds with a $\mathrm{SD}=2$, the calculated $z$ score is still 1.0 , but walk time was about $43 \%$ slower. If the timed 25 -foot walk test is used as a measure of clinical differences, scores from the actual performance data are more appropriate and informative for statistical comparisons, because the treatment effects would affect the magnitude of pre-post group means $(y, Y)$.

In summary, the present study has demonstrated the practice effects of the timed 25-foot walk test, which appears to stabilize after two practice trials. There are discrepancies within the current body of literature regarding the impact of the practice effect. However, it is unclear what the magnitude of its impact is owing to many investigators not clearly describing their testing procedures. Based on our results, participant familiarization with timed 25 -foot walk test could improve stability of walk performance scores in individuals with MS. Thus there is a need for standardized instructions and produces that researches adhere to. Our findings highlight new information relevant to administration of the timed 25-foot walk test; however, we appreciate limitations to our study, such as our limited sample. Our study also only tested participants with relapsing remitting MS with an EDSS score $<6.5$. Additional research is needed to specifically test across the different disease patters and with the inclusion of individuals with higher EDSS scores.

\section{Clinical messages}

- Baseline 25-foot ambulation tests should include at least two practice trials.

- Lack of familiarization with the 25-foot walk test can confound true performance improvements.

\section{Funding}

This research received no specific grant from any funding agency in the public, commercial, or not-for-profit sectors.

\section{References}

1. Kurtzke JF. Rating neurologic impairment in multiple sclerosis: an expanded disability status scale (EDSS). Neurology 1983; 33(11): 1444-1452.

2. Noseworthy JH. Clinical scoring methods for multiple sclerosis. Ann Neurol 1994; 36 Suppl: S80-85.

3. Hobart J, Freeman J and Thompson A. Kurtzke scales revisited: the application of psychometric methods to clinical intuition. Brain 2000; 123(Pt 5): 1027-1040.

4. Sharrack B, Hughes RA, Soudain S and Dunn G. The psychometric properties of clinical rating scales used in multiple sclerosis. Brain 1999; 122(Pt 1): 141-159.

5. Whitaker JN, McFarland HF, Rudge P and Reingold SC. Outcomes assessment in multiple sclerosis clinical trials: a critical analysis. Mult Scler 1995; 1(1): 37-47.

6. Kaufman M, Moyer D and Norton J. The significant change for the Timed 25-foot Walk in the multiple sclerosis functional composite. Mult Scler 2000; 6(4): 286-290.

7. Rudick R, Antel J, Confavreux C, et al. Recommendations from the National Multiple Sclerosis Society Clinical Outcomes Assessment Task Force. Ann Neurol 1997; 42(3): 379-382.

8. Rudick R, Antel J, Confavreux C, et al. Clinical outcomes assessment in multiple sclerosis. Ann Neurol 1996; 40(3): 469-479.

9. Fischer JS, Rudick RA, Cutter GR and Reingold SC. The Multiple Sclerosis Functional Composite Measure (MSFC): an integrated approach to MS clinical outcome assessment. National MS Society Clinical Outcomes Assessment Task Force. Mult Scler 1999; 5(4): 244-250.

10. Cutter GR, Baier ML, Rudick RA, et al. Development of a multiple sclerosis functional composite as a clinical trial outcome measure. Brain 1999; 122(Pt 5): 871-882.

11. Cohen JA, Cutter GR, Fischer JS, et al. Use of the multiple sclerosis functional composite as an outcome measure in a phase 3 clinical trial. Arch Neurol 2001; 58(6): 961-967.

12. Soilu-Hanninen M, Aivo J, Lindstrom BM, et al. A randomised, double blind, placebo controlled trial with vitamin D3 as an add on treatment to interferon beta-1b in patients 
with multiple sclerosis. J Neurol Neurosurg Psychiatry 2012; 83(5): 565-571.

13. Spain RI, St George RJ, Salarian A, et al. Body-worn motion sensors detect balance and gait deficits in people with multiple sclerosis who have normal walking speed. Gait Posture 2012; 35(4): 573-578.

14. Gijbels D, Dalgas U, Romberg A, et al. Which walking capacity tests to use in multiple sclerosis? A multicentre study providing the basis for a core set. Mult Scler 2012; 18(3): 364-371.

15. Broekmans T, Roelants M, Feys P, et al. Effects of longterm resistance training and simultaneous electro-stimulation on muscle strength and functional mobility in multiple sclerosis. Mult Scler 2011; 17(4): 468-477.

16. Bosma LV, Kragt JJ, Knol DL, Polman CH and Uitdehaag BM. Clinical scales in progressive MS: predicting longterm disability. Mult Scler 2012; 18(3): 345-350.

17. Phan-Ba R, Pace A, Calay P, et al. Comparison of the timed 25-foot and the 100-meter walk as performance measures in multiple sclerosis. Neurorehabil Neural Repair 2011; 25(7): 672-679.

18. Ratchford JN, Shore W, Hammond ER, et al. A pilot study of functional electrical stimulation cycling in progressive multiple sclerosis. Neuro Rehabilitation 2010; 27(2): 121-128.
19. Widener GL, Allen DD and Gibson-Horn C. Balance-based torso-weighting may enhance balance in persons with multiple sclerosis: preliminary evidence. Arch Phys Med Rehabil 2009; 90(4): 602-609.

20. Sutliff MH, Naft JM, Stough DK, Lee JC, Arrigain SS and Bethoux FA. Efficacy and safety of a hip flexion assist orthosis in ambulatory multiple sclerosis patients. Arch Phys Med Rehabil 2008; 89(8): 1611-1617.

21. Ytterberg C, Johansson S, Andersson M, Widen Holmqvist L and von Koch L. Variations in functioning and disability in multiple sclerosis. A two-year prospective study. J Neurol 2008; 255(7): 967-973.

22. Solari A, Radice D, Manneschi L, Motti L and Montanari E. The multiple sclerosis functional composite: different practice effects in the three test components. J Neurol Sci 2005; 228(1): 71-74.

23. Schwid SR, Goodman AD, McDermott MP, Bever CF and Cook SD. Quantitative functional measures in MS: what is a reliable change? Neurology 2002; 58(8): 1294-1296.

24. Rudick RA, Cutter G and Reingold S. The multiple sclerosis functional composite: a new clinical outcome measure for multiple sderosis trials. Mult Scler 2002; 8(5): 359-365.

25. Rosti-Otajarvi E, Hamalainen P, Koivisto K and Hokkanen L. The reliability of the MSFC and its components. Acta Neurol Scand 2008; 117(6): 421-427. 